Didáctica Geográfica n 21, 2020,pp. 19-39

DOI: https://doi.org/10.21138/DG.471

ISSN electrónico: 2174-6451

\title{
FORMAR PARA LA DOCENCIA EN GEOGRAFÍA: UN CAMINO PARA LA COOPERACIÓN
}

\author{
GEOGRAPHY TEACHER FORMATION: A PATH TOWARDS COOPERATION
}

FORMATION À L'ENSEIGNEMENT EN GÉOGRAPHIE: UNE VOIE DE COOPÉRATION

Lana de Souza Cavalcanti

Universidade Federal de Goiás, Brasil

ls.cavalcanti17@gmail.com

Leovan Alves Dos Santos

Universidade Federal de Goiás, Brasil

leovanalves@yahoo.com.br

Recibido: 21/05/2020

Aceptado 15/09/2020

\section{RESUMEN:}

Este trabajo presentará elementos de la formación continua del profesorado de Geografía, a partir de la composición de grupos cooperativos de profesores de esa asignatura. Destacaremos la centralidad del trabajo docente en tiempos de crisis y su relevante papel social para el desarrollo del pensamiento geográfico de los estudiantes. Por último, discutiremos las potencialidades y límites de las Comunidades de Práctica, presentando sus características, la concepción de cooperación y el camino metodológico de los grupos del Laboratório de Estudos e Pesquisa em Ensino de Geografia (LEPEG), de la Universidade Federal de Goiás, juntamente con la formación continua de profesores de Geografía. 


\section{Palabras Clave:}

Profesor de Geografía; formación de profesores; investigación colaborativa; pensamiento geográfico; comunidades de práctica.

\section{Abstract:}

In this paper we present some elements that involve the continued formation of geography teachers, which is possible due to the composition of collaborative groups. It highlights the centrality of teaching work in times of crisis and its relevant social role to the formation of students' geographical thought. This paper discusses potentialities and limits of Communities of Practice by presenting the characteristics and concepts of cooperation alongside the methodological path that orientates the Laboratório de Estudos e Pesquisa em Ensino de Geografia (LEPEG) at Universidade Federal de Goiás in its articulation with geography teachers' continuous formation.

\section{KEYWORDS:}

Geography teacher; teacher formation; collaborative research; geographical thought; communities of practice.

\section{RÉSUMÉ:}

Dans cet article, nous présenterons quelques éléments concernant la formation continue du professeur de Géographie, rendue possible par la composition des groupes coopératifs. Nous soulignerons la centralité du travail d'enseignement en temps de crise et son rôle social pertinent pour le développement de la pensée géographique chez les étudiants. Nous discuterons des potentialités et des limites des communautés de pratique, en présentant leurs caractéristiques, la conception de la coopération et le chemin méthodologique qui guide deux groupes du Laboratoire d'études et de recherche en enseignement de la géographie (LEPEG), de l'Université fédérale de Goiás, articulé à la formation continue des professeurs de géographie.

\section{MotS-CLÉS:}

Enseignant en Géographie; formation des enseignants; recherche collaborative; pensée géographique; communautés de pratique. 


\section{INTRODUCCIÓN}

El trabajo docente, consciente del concepto de Geografía y cómo ella sirve en la escuela, es una de las formas efectivas para que la enseñanza de esa asignatura atienda a las demandas sociales actuales. En ese contexto, el actuar docente necesita ser intencionado para que el aprendizaje de los alumnos sea posible. Uno de los pilares de la efectividad de este trabajo abarca los aspectos de la formación, inicial y continua del profesorado. Dicho esto, se comprende que la formación docente es un tema relevante para los investigadores en el área de la educación.

En el campo de la Geografía, la preocupación por ese tipo de formación viene, por lo que se puede deducir, principalmente del hecho de que, para una "comunidad" de profesores (de la escuela básica, en formación inicial, formadores de profesorado), los desafíos cotidianos para la actuación docente son diarios. Esos desafíos exigen que el profesor de geografía maneje diferentes estrategias para intervenir positivamente en el transcurso del desarrollo intelectual, social, afectivo de los estudiantes, independientemente de las interferencias externas a la dinámica de la escuela. Para hacer frente a ello se justifican, pues, inmersiones teórico-prácticas para la formación docente.

En consonancia con esas demandas puestas al profesor de Geografía en la escuela, expondremos, en este trabajo, elementos de la formación continua del profesor de Geografía, posibilitada por la composición de grupos cooperativos. Inicialmente, destacaremos la centralidad del trabajo docente en tiempos de crisis y su relevante rol social para la formación del pensamiento geográfico de los alumnos. Después, discutiremos las potencialidades y relacionando dos ejemplos de esos grupos en el campo de la formación continua del profesorado de Geografía en funcionamiento en el Laboratório de Estudos e Pesquisa em Ensino de Geografia (LEPEG), de la Universidade Federal de Goiás. En esta investigación resaltaremos sus características, la concepción de cooperación y el camino metodológico desarrollado en esas comunidades.

\section{LA CENTRALIDAD DEL PROFESOR EN TIEMPOS DE CRISIS}

Creemos que cualquier reflexión académica no puede dejar de realizar análisis coyunturales. Sin embargo, hay períodos en la historia en los cuales esos análisis se imponen con más fuerza, como es el caso del actual momento en que hay una tensión global provocada por una pandemia causada por el nuevo coronavirus, conocido oficialmente como COVID-19, en el año de 2020. Esta es una crisis de escala mundial con todo tipo de consecuencias, ya que es de origen sanitaria, pero tiene repercusiones en las dimensiones económicas, sociales, políticas, sociológicas, psicológicas y educativas.

En ese escenario pandémico, parece secundario discutir cuestiones como la que se propone este artículo: la formación docente en Geografía. No obstante, podemos 
argumentar que no es una cuestión menor. Justamente en momentos de crisis, nos parece que los profesores hacen una de las tareas más importantes en la sociedad y sus actuaciones pueden ser de gran contribución para la superación de algunas de las dificultades vivenciadas por la sociedad.

El trabajo de los profesores está dedicado al desarrollo de la inteligencia humana y a su formación. El profesor trabaja para el desarrollo de las personas, de su capacidad de comprender y de resolver los problemas que se instauran a lo largo de la historia en diferentes escalas. Esa relevancia no se altera en tiempos de crisis, al contrario, en esos momentos son más evidenciadas las demandas para el incremento de las capacidades humanas para el enfrentamiento de crisis. Ese desarrollo puede ser promovido por la actuación docente en los procesos de escolarización, por ello es más que pertinente hablar de formación docente, inicial y continua, en ese contexto.

Hay, efectivamente, una centralidad de la actuación docente cuando se quiere invertir en la calidad de los resultados de los procesos formativos en términos de desarrollo de la capacidad humana y de desarrollo amplio del alumnado, en todos los niveles de enseñanza. El trabajo del profesor es relevante, luego se le debe regalar la posibilidad de autonomía, fortaleciendo así su profesionalidad. Pero dar autonomía al docente no se trata de ponerlo como culpable de los resultados educativos, sino de propiciar condiciones para que se pueda atribuir al él parte de la responsabilidad del proceso de aprendizaje. Por lo tanto, durante los momentos de crisis social, de crisis planetaria, de crisis en la educación, se refuerza la necesidad de reflexionar acerca del trabajo docente y su relevante papel social. De la misma forma, se realza la vinculación de ese trabajo con una defensa de la educación centrada en el desarrollo humano amplio y en una sociedad justa y democrática.

El esfuerzo por un trabajo docente calificado acentúa la relevancia de invertir en los procesos formativos de los profesores, que es el tema de este artículo. Ya es consolidado entre los expertos del área la concepción de que el profesorado debe ser formado llevando a cabo principios generales fundamentados. El entendimiento es que la formación debe ser amplia, consistente y atender a las múltiples dimensiones y referencias del conocimiento: la ciencia de referencia, las ciencias de la educación, las ciencias de la sociedad.

Sobre el tema se puede destacar la contribución de Shulman (2005), que menciona las fuentes de conocimiento de base para la actuación del profesor: 1. formación académica, inicial y continua; 2. documentos oficiales, curriculum, textos didácticos; 3. investigaciones académicas, principalmente las que involucran la enseñanza y el aprendizaje, 4. práctica docente. La formación teórica y metodológica es una referencia importante que considerar en la actuación docente, pues se articula con otras en la composición de lo que el autor llama de "conocimiento pedagógico del contenido", que es, de hecho, la orientación más inmediata de su trabajo cotidiano. 
En ese sentido, nuestros estudios están vinculados a los retos de formar profesores basados en los principios de Shulman (2005), para que los docentes se reconozcan como intelectuales, autónomos y autores de sus trabajos y que ejerzan una profesión relevante para la sociedad. Ese entendimiento orienta acciones en los diferentes momentos de la formación inicial y continua del profesorado, en los cuales se tiene la oportunidad de reflexionar y problematizar su actuación, de analizarla a la luz de fundamentos teórico-conceptuales, de formular teorías y caminos y hacer ecuaciones para las situaciones-problemas evidenciadas.

En reuniones celebradas en grupos de colaboración y en cursos de capacitación surgen, con frecuencia, cuestionamientos generales del profesorado, como:

Mediante políticas educativas, como las que han sido implementadas en Brasil en los últimos años, ¿cómo puedo realizar mi trabajo?

¿Cómo puedo posicionarme frente a las imposiciones externas?

¿Qué autonomía tengo para desarrollar mi trabajo con autoría?

¿Qué orientaciones pedagógico-didácticas y curriculares debo seguir? ¿La de los dirigentes y gestores de la educación o la de especialistas del área?

Cuando hay interferencias externas que provocan rupturas en el trabajo que realizo con base en mi experiencia y mis creencias, ¿cómo debo hacer/reaccionar?

Entre los formadores de profesores (en el contexto de las universidades), también son frecuentes cuestionamientos semejantes:

¿Se debe (o es posible) reafirmar, en las prácticas formativas, los principios de autonomía y autoría del trabajo docente?

¿Qué es autonomía docente? y ¿cuál es su límite en la práctica?, ¿cuál es la contribución de la formación teórica consistente para hacer efectiva la autonomía? ¿Qué es ser autor del trabajo docente? ¿quédimensión la autoría docente se manifiesta? Frente a políticas educacionales más directivas, ¿es posible y adecuado desarrollar propuestas de trabajo con cierto grado de autonomía y autoría docentes?

En este contexto, permanece la proposición de fortalecer las rutas de la formación docente, inicial y continua como una vía importante para enfrentar los diferentes contextos que surjan a lo largo del ejercicio profesional del profesor. Así, algunos cuestionamientos más generales orientan nuestro trabajo de investigación que se inclina justamente a encontrar caminos por los cuales esa formación pueda ser más consistente y de buena calidad. Algunos de las preguntas que abarcan la búsqueda de esas rutas son: 
¿Cómo formar profesores para enfrentar los diversos desafíos de su realidad profesional en diferentes contextos?

¿Cómo formar profesores de Geografía con condiciones intelectuales que fundamenten la defensa de un trabajo autónomo y con autoría?

¿Cómo proveerlos de conocimientos, convicciones y condiciones cognitivas operativas para la actuación profesional?

¿Qué tipo de conocimiento compone el conocimiento docente?

¿Cómo ocurre el desarrollo cognitivo del profesor?, ¿cómo él aprende a enseñar?

Los caminos formativos realizados en nuestras instituciones, en las últimas décadas ¿han sido adecuados?, ¿cuáles son esos caminos?

Esas indagaciones son consideradas relevantes para comprender los procesos formativos de los profesores y sus actuaciones profesionales en las diferentes realidades educativas. El conjunto de cuestiones presentadas ha dirigido nuestras investigaciones en el sentido de encontrar modos más seguros de actuación profesional de profesores formadores, futuros profesores y profesores de la educación básica de la asignatura de Geografía, a fin de facilitar el aprendizaje significativo en la educación básica. Nuestra opinión es la de que la formación docente tiene un carácter general, con aspectos comunes al conjunto de profesores, pero también tiene peculiaridades para el profesor de Geografía, que ponen objetivos y metas específicas para su formación y consecuentemente para la actuación de ese profesional. Los objetivos y metas de ese profesor serán el tema de la siguiente sección.

\section{EL PROFESOR DE GEOGRAFÍA Y SU ROL SOCIAL: SU ESPECIFICIDAD EN EL DESARROLLO DE LOS ALUMNOS}

En esta investigación, algunas referencias son usadas para reafirmar el relevante rol de la Geografía en la formación básica de los ciudadanos. Esa ciencia ha producido, a lo largo de la historia, conocimiento sobre la espacialidad de los fenómenos. Con base en principios, categorías de análisis y lenguaje especializada, ella ha producido teorías y reflexiones que expresan una visión particular sobre el mundo desde su institucionalización como ciencia en el siglo XIX. Según Gomes (2013), la Geografía es una forma de inteligencia, un modo de pensar sobre el mundo, una cierta manera de mirar las cosas.

Más allá de ese modo especializado y sistemático de producir lecturas y análisis consistentes que "explican" el mundo, la Geografía también capta una dimensión de la dinámica de espacialidad mundial. Esa ciencia entiende que las personas en general participan de la producción de esa espacialidad, o sea, están en ella involucradas. Sus prácticas cotidianas están condicionadas por espacialidades ya producidas y, por su dinámica, resultan en nuevas espacialidades. Así que hay un movimiento dialéctico en el cual las personas y sus prácticas espaciales están en interrelación e interdependencia. 
Por ende, conocer muy bien la espacialidad como dimensión inherente a la realidad en que viven interesa a los alumnos. El objetivo es que, al conocerla, ellos puedan comprender su significado en sus vidas y en la vida de la sociedad de modo general. Ese conocimiento, posible por el desarrollo del pensamiento geográfico, a su vez, fundamenta las posibilidades de acciones ciudadanas de los alumnos. Reaccionar de manera ciudadana a partir del conocimiento de la espacialidad es, justamente, la tarea de la Geografía en la escuela, es decir, su razón de existir como disciplina en la formación básica de niños y jóvenes en su proceso de escolarización.

Al seguir esta línea de argumentación, es importante aclarar el entendimiento del pensamiento geográfico como objetivo de la enseñanza de Geografía. El pensamiento geográfico es una habilidad teórico-conceptual fundamental de la formación humana, ya que permite una relación calificada del sujeto y el mundo, una relación con abundante mediación de elementos que le permite comprender la complejidad de este mundo en su espacialidad. Ese modo de pensar tiene, en primer lugar, el cuidado con la localización de los fenómenos y su distribución, pero también indaga sobre el fraccionamiento de acciones de la localización/distribución, porque quiere comprender su significado.

El pensamiento geográfico está interesado en las implicaciones que ese tipo de fenómeno localizado tiene: ¿por qué está ahí? ¿qué significa estar en ese lugar? ¿dónde más está? ¿qué provoca su localización en ese lugar? ¿qué llevó a la localización en ese lugar? Las respuestas a esas preguntas son dadas a partir de un tipo de razonamiento, que puede también ser llamado de raciocinio geográfico. El raciocinio geográfico es la operacionalización del pensamiento, un modo de manejar ese pensamiento, que se inicia con saber localizarse y sigue con la identificación de las distribuciones, de las posiciones, de las escalas y conexiones (Moreira, 2007).

Para que la enseñanza de Geografía sea efectiva, se puede afirmar que el trabajo docente consiste en dar subsidios para construir el pensamiento geográfico del estudiante por medio de los contenidos escolares. Esa afirmación implica ser contario a la enseñanza direccionada a la mera transmisión de contenidos, ya que la perspectiva tradicional de enseñanza no puede figurar como meta de cumplimiento obligatorio de programas estipulados a priori. La enseñanza no tradicional, más que la presentación y disposición de contenidos geográficos, invertirá en acciones que tengan como resultado el desarrollo de los alumnos, principalmente cuanto al pensamiento geográfico.

Para el desarrollo de ese pensamiento, hay que destacar la formación de conceptos. La concepción que orienta nuestro trabajo es la de que el pensamiento es compuesto por la articulación de racionamientos, lenguajes y conceptos que son/pueden ser movilizados en la relación activa (que comprende, que analiza, que actúa) del sujeto con el mundo. Entre los conceptos destacados más genéricos están: paisaje, lugar y territorio (Cavalcanti, 2019). 
En este momento, retomamos el tema de la formación docente en Geografía, que presupone proporcionar orientaciones básicas para el trabajo de mediación para el desarrollo del pensamiento geográfico del alumno, trabajo que requiere un perfil profesional a ser formado. La formación docente puede ser guiada por algunos principios generales, entre ellos están: autoformación y formación continua; interdisciplinaridad; interrelación entre teoría y práctica; relación indisoluble entre enseñanza e investigación. En el caso específico de profesores de Geografía, entendemos que el principio de la formación de conocimiento consistente de la Geografía es uno de los más importantes.

Ese conocimiento trata de la historia de esa ciencia, sus proposiciones para contribuir con la sociedad a lo largo de la historia, su producción, sus teorías, los resultados de esas teorías en términos de conocimiento de mundo y de la espacialidad del mundo. Además, es necesario que el profesor sepa de modo muy especial el significado de esa ciencia para la humanidad, para la formación de las personas. En ese sentido, los profesionales del área deben poder problematizar los sentidos y significados de esa ciencia en la escuela y cuestionar cómo los sujetos aprenden o pueden aprender los contenidos de la Geografía. Mejor dicho, es importante que el profesor aprenda Geografía en sus momentos de formación, pero también que aprenda cómo enseñar la asignatura y qué problematización existe sobre la Geografía escolar (Cavalcanti, 2017).

Comprendemos que ello es una posibilidad para que los profesores puedan actuar, con autoría y autonomía, orientados por el propósito de contribuir para la formación del pensamiento geográfico de los alumnos. Sin embargo, esa posibilidad no está garantizada en la realización de las prácticas formativas, en proyectos de formación inicial o continua. Justo lo contrario, es construida en un complejo proceso de desarrollo profesional, que involucra más que prácticas escolares generales, pero también la reflexión y la metacognición de los profesionales de la educación.

Así, hay una potencialidad en las prácticas formativas en las que profesores colectivamente cuestionan la realidad que viven y piensan en estrategias para enfrentar sus dificultades con la finalidad de lograr aprendizajes significativos con relación al desarrollo del pensamiento geográfico. La convicción de esa potencia de trabajo colectivo y colaborativo de profesores de Geografía es una importante inversión para la realización de diversos trabajos en el ámbito del Laboratório de Estudos e Pesquisa em Ensino de Geografía (LEPEG), del Curso de Geografia de Instituto de Estudos SocioAmbientais, de la Universidade Federal de Goiás. Entre esos trabajos, vamos a abordar algunos de ellos, a seguir. 


\section{ELDESARROLLO PROFESIONALEN FOCO: PESQUISAS Y PROPUESTAS DE COLABORACIÓN EN EL CAMPO DE LA GEOGRAFÍA}

Para ampliar las posibilidades formativas del profesor, entendemos que el concepto de desarrollo profesional (Imbernón, 2010) es fecundo e instiga bastante, pues se refiere a toda acción sistemática que debe ser un proceso continuo y que busca mejorar la práctica, las creencias y los conocimientos profesionales. Esa concepción ha fundamentado nuestra actuación en el sentido de investigar sobre el trabajo docente en Geografía y sus potencialidades para el aprendizaje de los alumnos. Con base en ella, ha sido definida la investigación colaborativa como principal camino metodológico de los trabajos que realizamos en el LEPEG.

La investigación colaborativa es un conjunto de posibilidades de desarrollo metodológico de investigación y de formación docente. Ella está pautada en la colaboración entre diferentes sujetos del proceso educativos: profesores de la red básica de enseñanza, profesores en formación (alumnos de graduación y postgrado) y profesores formadores (universitarios). La defensa de la necesidad de aproximación entre universidades y escuelas básicas, para efectuar políticas y programas de mejora en la educación, y la concepción de desarrollo profesional continuo de profesores ha reforzado la creencia en la potencia de constitución de grupos de profesores en situación y contextos diferentes de ese desarrollo, como prácticas formativas. Esos grupos pueden ser entendidos como Comunidades de Práctica.

Según nuestro entendimiento, una Comunidad de Práctica $(\mathrm{CoP})$ se refiere al proceso de colaboración, de acción, de compartir conocimientos, de intereses, de actividades y prácticas para la producción de conocimiento personal y colectivo de los profesores. En una CoP, es posible realizar el estudio y la reflexión de los modos de enseñar y aprender, lo que puede contribuir significativamente para el desarrollo profesional continuo del profesor.

Así, actuar para el buen desarrollo de una CoP está asociado al desencadenamiento de procesos de reflexión compartida y al establecimiento de un ambiente cooperativo, de diálogo abierto y de confianza. En ese ambiente, las acciones son coordinadas, planificadas y negociadas conjuntamente, buscando un desarrollo profesional, personal y colectivo. Esos principios orientan nuestra actuación en los trabajos que hemos realizado, algunos de los cuales serán descriptos en la próxima sección. Antes, es importante aclarar todavía algunas características de ese tipo de práctica formativa, conforme las referencias del área.

Una de las características de los grupos colaborativos es destacada por Fiorentini (2010a). El autor resalta que el estudio y la formación docente tienen dimensiones que involucran la dedicación en comprender los modos de enseñar y de aprender en las escuelas. Esos grupos constituyen comunidades investigativas y/o reflexivas, formadas 
por profesores de la escuela, profesores/investigadores de la universidad y futuros profesores que estudian, comparten, investigan, discuten y escriben sobre la práctica pedagógica en las escuelas en un ambiente colaborativo.

El concepto de comunidad de práctica tiene la dimensión de aprendizaje como actuación social y no individual. Las CoPs, en ese sentido, se han constituido como una alternativa para el desarrollo profesional de profesores y para la producción de un repertorio de prácticas educativas fundamentadas en investigaciones sobre la práctica de enseñar y aprender.

La constitución de una CoP posibilita la construcción de una instancia por medio de la cual los profesores piensan, problematizan, actúan y reflexionan en colaboración sobre aquello que hacen y sobre la forma como manejan su proceso de enseñanza con los contenidos escolares. Por ser un grupo, cada uno de los profesores busca fortalecerse con la cooperación de los otros integrantes. El profesor, al relatar sus experiencias y dilemas en el grupo, aprende con los otros participantes y les enseña, incluso, cómo hacer sus proprios relatos. Fiorentini (2010b) destaca que el profesor aprende al narrar, ya que organizar sus ideas y, al sistematizar sus experiencias, produce nuevos significados. El profesor también enseña al grupo cuando (re)significa los saberes y experiencias de los otros profesores por medio de sus narrativas.

Para la formación de un grupo cooperativo que discute y reflexiona en conjunto sobre su práctica, no se puede desconsiderar la necesidad de una serie de factores estructurales favorables a las condiciones materiales y de trabajo - jornadas de trabajo reducidas que posibiliten al profesor buscar su propio desarrollo profesional, clases menos numerosas para que el profesor pueda ejercer prácticas innovadoras, reconocimiento de los grupos de estudio dentro de las escuelas como prácticas de formación continua, mejor sueldo para participación en eventos, compra de libros y otras condiciones. Esas condiciones, sin embargo, han sido, casi en su totalidad, negadas por las instituciones y por las políticas públicas brasileñas fuertemente influenciadas por los modelos neoliberales.

En ese contexto adverso al desarrollo profesional continuo y con calidad, es posible identificar grupos que resisten al trabajo repetitivo, sin reflexión y buscan caminos para la cooperación y para su desarrollo formativo. Los profesores que poseen una postura de estudio y buscan compañeros para analizar y discutir sus prácticas pedagógicas presentan fuertes indicios de que desean una participación más activa, continua y autónoma en los proyectos formativos de las escuelas donde actúan. Ese tipo de profesional, frecuentemente, se maneja como protagonista en los procesos de cambio curricular de la escuela y de su proprio proceso de desarrollo profesional, especificando las prioridades de estudios compartidos y reflexionando sobre la propia práctica de enseñar y aprender los diferentes componentes curriculares de la escuela. 
Montero (2001), en ese sentido, destaca que la preocupación por la construcción del conocimiento en la enseñanza, la transformación de ello en conocimiento profesional de los profesores y, en particular, la reivindicación del conocimiento generado por los profesores por medio del análisis de su práctica han sido, en las últimas décadas, cuestiones claves de la investigación didáctica. La evolución que se ha verificado en la investigación sobre la enseñanza, contemplando temas variados - desde el interés por los comportamientos de los profesores (la actuación en sus clases) hasta el interés por los conocimientos, creencias, sentimientos, emociones, que están junto a su actividad profesional - hizo que esas cuestiones se volvieran prioritarias. De ahí, la necesidad de examinar, de nuevo, las relaciones entre la investigación sobre la enseñanza y la formación de profesores.

El concepto de Comunidad de Práctica, ciertamente, no es algo nuevo. Sin embargo, solo recientemente esas ideas teóricas han sido puestas como objeto de estudio de diversas áreas, incluso en el área de la enseñanza de Geografía. Las comunidades de práctica se han constituido, en los últimos años, en un espacio promisor para el desarrollo de investigaciones que busquen comprender los procesos formativos de grupos de profesores y futuros profesores.

En un levantamiento, realizado en las plataformas que son referencias en citas científicas, Scopus, Web of Science y Scielo, fue posible constatar la poca presencia de investigaciones en esa modalidad publicadas sobre el trabajo colaborativo docente en Brasil.

La tabla siguiente permite evidenciar los trabajos:

\begin{tabular}{cccc}
\hline \multicolumn{4}{c}{ Plataforma Scopus } \\
\hline Palabra clave & $\begin{array}{c}\text { Trabajos } \\
\text { identificados } \\
\text { a partir de la } \\
\text { palabra clave }\end{array}$ & $\begin{array}{c}\text { Trabajos seleccionados } \\
\text { (relacionados a la } \\
\text { enseñanza y formación de } \\
\text { profesores) }\end{array}$ & $\begin{array}{c}\text { Temática } \\
\text { relacionada con } \\
\text { enseñanza de } \\
\text { Geografía }\end{array}$ \\
Grupo de Profesores & 65 & 4 & 0 \\
Comunidad de práctica & 142 & 11 & 0 \\
Trabajo colaborativo & 200 & 5 & 0 \\
\hline & Plataforma Web of Science & 0 \\
\hline Trabajo colaborativo & 116 & 5 & 0 \\
\hline Grupo de Profesores & 0 & 0 & 0 \\
Comunidad de práctica & 11 & 5 & 0 \\
Grupos de aprendizaje & 3 & 3 & 0 \\
Colaboración docente & 2 & 1 & $\mathbf{0}$ \\
\hline Total & $\mathbf{5 3 9}$ & $\mathbf{3 4}$ & \\
\hline
\end{tabular}

TABLA 1. Levantamiento realizado en plataformas referenciales de citaciones científicas sobre trabajo colaborativo docente en el Brasil (2006-2019). Fuente: Elaboración propia (2020). 
Conforme lo observado en la tabla 1, fueron identificados, en el universo de 539 estudios publicados sobre formación de profesores, solamente 34 trabajos que versan sobre esa formación docente de forma colaborativa. De esa forma, se percibe una laguna en el desarrollo de comunidades de práctica en diferentes áreas, para que puedan ser establecidas con más vigor prácticas cooperativas que resulten en alteraciones de la dinámica curricular de las escuelas, garantizando, así, la inclusión, la participación y el aprendizaje de todos los estudiantes.

En este levantamiento, se pudo percibir que existen trabajos relacionados con la formación colaborativa y continua de profesores principalmente en Matemática, Química y Biología. Otro dato que merece ser destacado es el hecho de no haber sido identificadas publicaciones que investiguen el trabajo colaborativo de los docentes y la enseñanza de Geografía en Brasil. Por eso, se infiere que hay una demanda referente al desarrollo más sistemático de investigaciones que involucren las potencialidades del trabajo colaborativo en la formación inicial y continua de profesores de Geografía.

Tanaka y Passos (2017) destacan que los grupos cooperativos se constituyen en terreno fértil para la aproximación de profesores de diversas escuelas, con diferentes períodos de experiencia en el aula, en la dirección del desarrollo profesional de todos los involucrados. Eso pasa, porque, las prácticas con enfoque en el aprendizaje de los estudiantes constituyen uno de los pilares de los cambios. En la ejecución de las actividades formativas en los grupos cooperativos es importante la apertura y la confianza. Para ello, es necesario que todos estén en un mismo nivel y que todos se sientan partícipes en equivalencia, estableciéndose una relación de carácter horizontal. Aunque existan diferentes niveles de formación, de experiencia profesional, de tiempo de carrera como profesor; todos necesitan buscar un lugar en común dentro del grupo, contando con la participación y con el interés particular de cada uno.

\section{GRUPOS COPERATIVOS DE PROFESORES DE GEOGRAFÍA EN EL LEPEG/UFG: UN CAMINO PARA LA FORMACIÓN CONTINUA}

Conforme lo destacado en la tabla 1 del apartado anterior, hay evidencias de pocas investigaciones sobre el trabajo cooperativo en la formación continua del profesorado en Brasil. En el levantamiento realizado, identificamos dos grupos de profesionales que enseñan matemáticas que siguen esa metodología hace más tiempo y que poseen una sistematización más consolidada.

El primer grupo está compuesto por profesores de matemáticas, alumnos de graduación, de postgrado y formadores de profesores, conducido por el profesor Dário Fiorentini (Fiorentini, 2010b) de la Unicamp (ciudad de Campinas/São Paulo), llamado Grupo de Sábado. El segundo grupo es el proyecto Rede colaborativa de 
práticas na formação de professores que ensinam matemática: múltiplos olhares, diálogos e contexto, una colaboración entre la Universidade Federal de São Carlos (UFSCar), campus Sorocaba (São Paulo), la Pontifícia Universidade Católica de São Paulo (PUC/SP) y la Universidade Federal do ABC (UFABC). La profesora Ana Lúcia Manrique, de la PUC/SP, es una de las coordinadoras y el grupo es desarrollado en São Paulo, en el ámbito del Programa Observatório da Educação (OBEDUC), de la Capes (Manrique, 2017).

Sobre enseñanza de Geografía en Brasil, destacamos en este texto dos grupos recién creados (en 2019), de los cuales participamos, y que son ejemplos de propuestas de cooperación entre profesores en formación inicial y continuada. El primer grupo se refiere a la Rede Colaborativa de Ensino da Cidade e as Cidadanias (RECCI). Esa red está ligada al proyecto Desenvolvimento Profissional do Professor de Geografia - Grupo de Discussão/Formação, conducido por la profesora Lana de Souza Cavalcanti, de la Universidade Federal de Goiás - UFG. Su objetivo es investigar aspectos relacionados con el desarrollo profesional de profesores de Geografía, con protagonismo para la construcción de las bases del conocimiento profesional y del pensamiento geográfico, en situaciones prácticas, con la enseñanza enfocada en la vida urbana ciudadana, en el contexto del territorio de Goiás.

El segundo grupo colaborativo es el Grupo de Discussão (GD) em geopolítica, coordinado por Leovan Alves dos Santos, como conducción metodológica de la tesis de doctorado titulada $O$ professor de Geografia do Ensino Médio e as orientações curriculares: o conteúdo geopolítica nas propostas recentes, junto al Programa de Pós-Graduação em Geografia da Universidade Federal de Goiás (UFG). El grupo está compuesto por profesores de Geografía que actúan en la red estadual de Goiás/ Brasil. El énfasis del grupo es la perspectiva de pensar sobre la práctica de la clase, los desafíos que el profesor enfrenta para llevar a cabo sus propuestas de enseñanza con el contenido de geopolítica en el contexto de cambios de orientaciones curriculares que son propuestas por las normativas nacionales y locales. En ese sentido, el grupo busca discutir las perspectivas y las posibilidades de los profesores frente a las orientaciones y prescripciones curriculares para la planificación y desarrollo de las clases.

A continuación, presentamos algunos aspectos de los grupos:

\subsection{Rede Colaborativa de Ensino da Cidade e as Cidadanias - RECCI}

RECCI es un grupo constituido en 2019, compuesto por formadores de profesores de Geografía de diferentes instituciones de Goiás, la Universidade Federal de Goiás, en Goiânia y la Universidade Federal de Jataí, en Jataí; alumnos de la graduación y del postgrado de las instituciones y profesores de Geografía de Red Pública, Red 
Particular de Goiás y Red Municipal. En el grupo participan diferentes profesores: en formación inicial, en ejercicio profesional y profesores formadores. Para su ejecución, son realizadas reuniones periódicas mensuales conforme disponibilidad de sus miembros:

\begin{tabular}{|c|c|}
\hline Categoría & Número \\
\hline Profesor entrenador & 6 \\
\hline Profesor de educación básica & 5 \\
\hline $\begin{array}{l}\text { Profesor de la Red de Educación Básica y Estudiante de Postgrado (maestría y } \\
\text { doctorado) }\end{array}$ & 7 \\
\hline Estudiante de graduación & 2 \\
\hline Total & 20 \\
\hline
\end{tabular}

Tabla 2. Componentes del Grupo de Rede de Ensino da Cidade e Cidadanias. Fuente: Cavalcanti (2020).

El grupo posee un tema central específico: la formación del profesor de Geografía que actúa para promocionar la vida urbana ciudadana. En ese sentido, se busca reflexionar sobre las demandas de la profesionalización docente frente al contexto brasileño actual. El profesor es llamado a comprender las tareas exigidas por su rol social, teniendo en cuenta una propuesta de enseñanza de Geografía enfocada en la formación ciudadana. Durante los encuentros, existe la posibilidad de varios procedimientos, pero, centralmente, el grupo se propone a realizar acciones para atender a las demandas propuestas por el propio grupo. Luego, el trabajo desarrollado tiene como orientador de discusiones el conjunto de categorías básicas del análisis del conocimiento y la práctica del profesor.

La dinámica de las reuniones en el Grupo es definida y preparada por sus propios miembros y se orienta por categorías como: ciudad y vida urbana, ciudadanía, conocimiento pedagógico del contenido y pensamiento geográfico. Además, son desarrollados recursos metodológicos expresados en actividades de problematización, sistematización y síntesis de contenidos (Cavalcanti, 2014), que no son utilizadas como orientación metodológica rígida a ser seguida, sino como propuesta para debate.

Cada reunión tiene los debates basados en un texto o fragmento de texto que aborda temas relacionados a las categorías ya señaladas y ejemplos de prácticas docentes relacionadas: 
Texto

Los fines y los contenidos de enseñanza

Formação de Professores: para uma Mudança Educativa

Cidadania no Brasil: o Longo Caminho

Por uma Geografia Cidadã: por uma Epistemologia da Existência Santos (1996)
Autor

Díaz; Porlán; Navarro (2017)

Garcia (1999)

Carvalho (2002)

TABLA 3. Textos estudiados en las reuniones de formación. Fuente: Elaboración propia (2020).

Las discusiones propuestas y la teoría contribuyen aún más a la profesionalización de los docentes, ya que son temas relevantes que repercuten directamente en la práctica pedagógica de los miembros. Además, complementar la capacitación para maestros es muy importante para que podamos enseñar teniendo como meta la educación ciudadana de nuestros estudiantes:

\begin{tabular}{cc}
\hline \multicolumn{2}{c}{ Temas discutidos en las reuniones - basados en la lectura de textos } \\
\hline $\begin{array}{c}\text { Principales desafíos en la práctica docente. } \\
\text { Mapa de contenido en la elaboración de } \\
\text { planes de enseñanza. }\end{array}$ & Desarrollo profesional docente. \\
\hline
\end{tabular}

\section{Actividades realizadas durante y entre reuniones}

Lectura, discusión y corrección para actualizar material didáctico para reimpresión. Fascículo didáctico: "Espacio urbano de la Región Metropolitana de Goiânia".

Lectura y discusión de textos de referencia sobre el tema.

Preparación y presentación en pequeños grupos de propuestas de mapas de contenido, centrados en el tema de la ciudad y los problemas urbanos.

Participación en taller, en la ciudad de Jataí, sobre el trabajo docente con el tema didáctico: relacionamiento entre la ciudad y el campo en Goiás

Preparación de una propuesta preliminar para un Programa de Educación Continua para profesores de Geografía con un enfoque en la enseñanza de la ciudad y la ciudadanía.

TABla 4. Temas y actividades desarrollados en las reuniones del Grupo de Rede de Ensino da Cidade e Cidadanias Fuente: Cavalcanti (2020).

Otro aspecto considerado relevante para este texto son las respuestas de los participantes sobre la contribución del grupo para el concepto de ciudadanía y la vida ciudadana urbana. También, los componentes del grupo participan de propuestas didácticas que tienen como objetivo integrar las orientaciones metodológicas ya referidas a sus prácticas docentes. Al final de una reunión, o conjunto de reuniones, 
los participantes hacen narrativas sobre sus experiencias en las actividades del grupo. Por fin, el conjunto de las discusiones, las propuestas didácticas y las narrativas son herramientas de análisis para la identificación de aspectos de los conocimientos de los profesores de Geografía y de su desarrollo profesional.

\subsection{Grupo de Discussão (GD) en Geopolítica}

La realidad del contexto escolar brasileño presenta dimensiones de trabajo al profesor que muchas veces dificultan las posibilidades de avanzar en su aprendizaje. Pese a los esfuerzos para lograr una formación continua que contribuiría para el desarrollo profesional docente, muchos profesores aún trabajan de forma aislada la mayor parte del tiempo. En muchas escuelas, está previsto en el calendario escolar encuentros de grupos de trabajo colectivo de profesores, pero, en la mayoría de las veces, los gestores destinan ese espacio para hablar sobre cuestiones burocráticas, tratando con negligencia cuestiones como la formación del profesor y las reflexiones sobre la práctica de enseñanza.

En ese escenario, Tanaka y Passos (2017) resaltan que solo vivir el cotidiano escolar no significa tener una formación continuada, ya que el simple hecho de participar de esa rutina no garantiza al profesor elementos para avanzar en su aprendizaje. Para que ello ocurra, necesariamente el profesor necesita compartir las prácticas que realiza en su cotidiano y reflexionar sobre ellas, para avanzar en su profesionalidad. Pensando en ese tipo de contexto, se ha constituido el Grupo de Discussão em geopolítica-GD, en 2018, como un proceso de construcción colectiva en que se volvió referencia la búsqueda de alternativas de trabajo conjunto (posibles proyectos de estudio, innovaciones en aula e investigación sobre la geopolítica y formas de enseñarla).

Son puntos comunes para investigación en el grupo:

- La búsqueda de conocimiento mutuo: para conocer qué saben, piensan, sienten y hacen los otros profesores del grupo sobre la enseñanza de Geografía, específicamente los caminos que cada uno hace al enseñar geopolítica.

- El cambio de experiencias y reflexiones sobre la enseñanza, en las escuelas, de Geografía en general y geopolítica en particular.

- El entendimiento sobre la necesidad de desarrollar el contenido de geopolítica en las clases.

- La compresión sobre por qué se trabaja determinado contenido y no otro, y sobre la orientación curricular que cada profesor sigue para su clase, al trabajar el contenido de geopolítica.

- La profundización teórico-conceptual y didáctico-pedagógico sobre la enseñanza de geopolítica. 
- La coordinación del grupo endiente que, cuando el docente avanza en su profesionalidad, él comprende la actividad de enseñar. Eso, por su vez, exige que los profesores busquen formas de desarrollo profesional a lo largo de toda la carrera. Por lo tanto, el GD en geopolítica es un espacio de formación y de constitución profesional del profesor y de construcción de su identidad. Ese bagaje permite a los profesores construir un conocimiento profesional que tiene la dimensión crítica, que es una importante dimensión para la autonomía profesional, como uno de sus componentes.

Un gran desafío del GD es la manutención del pensamiento de que nuestra actividad docente es importante. Al identificarnos como grupo docente/comunidad profesional que confía en sus pares y creer en la importancia de nuestro trabajo, estamos de acuerdo con Wenger (2006) apud Ferreira y Silva (2014), que defiende tres características importantes para la formación de una CoP: el dominio, la comunidad y la práctica.

El tema de interés compartido por los miembros es la geopolítica. A través del tema, se firmó un compromiso entre los profesores sobre cómo el contenido debe ser abordado. En ese GD, se busca estudiar y reflexionar sobre los contenidos de geopolítica teniendo como base su fuerte presencia en la enseñanza media y en los exámenes en larga escala, como, por ejemplo, el Exame Nacional do Ensino Médio (ENEM) en Brasil y las trasformaciones curriculares que están ocurriendo paralelamente en la red de enseñanza del Estado Goiás.

Involucrarse corresponde a la característica de comunidad, lo que hace esa práctica un importante aspecto a ser construido en el grupo. En ese sentido, los profesores realizan actividades conjuntas, comparten informaciones y cooperan entre sí: el GD es el espacio donde los docentes pueden hablar abiertamente sin críticas a una eventual falta de conocimiento de determinada dimensión del contenido o didáctico-pedagógica. También, el grupo es un espacio para buscar formas de corrección de una determinada laguna en la formación del profesor.

Además, las actividades que son desarrolladas en la comunidad contribuyen con la reflexión y la práctica en clase, componiendo así una tercera característica para la composición de una CoP. Los miembros del GD son practicantes al ejecutar en clase las propuestas que fueron discutidas en el grupo, al compartir las necesidades formativas, los recursos, las experiencias, las herramientas y las rutinas.

Los docentes figuran en las reuniones de GD con sus principales desafíos para enseñar elementos de Geografía, que pasan por la educación continua del profesorado, el tratamiento dado al contenido geográfico, la necesidad de despertar el interés del estudiante. Hay elementos que impregnan la dimensión de la motivación del profesor, su creencia y su forma de enseñar:

Mantener el interés de los estudiantes en el contenido que se trabaja: hay muchos proyectos que "florecen" y reducen la carga de trabajo del docente. No se pueden 
reestructurar las clases, un privilegio solo para las asignaturas de Lengua y Matemáticas. Es necesario superar la descripción y la memorización, la necesidad de que los estudiantes reconozcan la realidad en la que se insertan y la necesidad de aprender qué es geográfico. (Profesor I)

Hay un gran reto relacionado cómo le mostraré a mi estudiante que su realidad no es la realidad de todos. Hay un desafío para discutir la desigualdad. Comprender que hay problemas importantes en el mundo y que estos deben abordarse. Hay una dificultad en la enseñanza de los contenidos en el sentido de que el alumno de la periferia comprenda las características que involucran a la clase rica. (Profesor II) La comprensión del mundo por parte del alumno, entendiendo más allá del lugar donde viven y estudiantes de la periferia que no entienden más allá de este lugar. Además, tengo dificultades en cómo abordaré ciertos contenidos frente a esta realidad. (Profesor III)

El profesor necesita saber el contenido. Para mí, uno de los desafíos es el contexto pedagógico, el placer, la tendencia pedagógica y lúdica. Hay una dificultad para que el docente asuma la planificación de la lección. Creo que el profesor necesita ser diversificado, debe asumir la planificación, trabajar con metodologías, especialmente con estudiantes de secundaria, que no hacen que las clases sean aburridas. (Profesor IV)

Hay innumerables dificultades: falta de material; libro de texto que no "coincide" con el plan de estudios; falta de conciencia del equipo escolar por la disciplina de Geografía; pequeña carga de trabajo en la escuela secundaria; importancia de relacionar la teoría con la práctica. (Profesor V)

A partir de los testimonios, es posible afirmar que el GD auxilia la profesionalización docente. El énfasis del grupo es el desarrollo profesional pautado en la reflexión teórico-conceptual sobre el contenido de geopolítica y la relación que los profesores establecen con el curriculum prescrito al elaborar su propuesta didáctica y sobre cómo se hace efectiva esa propuesta en clase. El objetivo del GD es crecer profesionalmente al profundizar los estudios y propiciar, como consecuencia, mayor aprendizaje. Se trata así de un grupo de profesores preocupados con la enseñanza de Geografía y con el direccionamiento que están dando al contenido de geopolítica en la enseñanza media en Goiás.

\section{CONSIDERACIONES FINALES}

El desarrollo profesional docente es un proceso continuo, sistemático, dinámico; y ocurre a lo largo de la carrera profesional, en diversos momentos. Por eso, la formación 
inicial y continuada del profesor debe ser amplia, consistente y atender a las múltiples dimensiones y referencias del conocimiento: la ciencia de referencia, las ciencias de la educación y los conocimientos de la sociedad.

La reflexión sobre el conocimiento profesional docente debe estar presente en la acción de los profesores que forman otros profesores. Esas son cuestiones pertinentes para quienes tienen la responsabilidad de direccionar procesos sistemáticos de formación de los docentes en un área específica. Para que haya construcción de los conocimientos del profesor, en sus distintas dimensiones, que oriente una práctica reflexiva y constantemente creativa, es fundamental que los profesores formadores ejerciten en sus rutinas entre la docencia y la investigación científica.

Los profesores participantes de los grupos cooperativos aquí ejemplificados se aproximan por intereses comunes de formación continua, por sus vínculos en la propia red de educación, por las mediaciones simbólicas por ellos interiorizadas, por la necesidad de despertar el interés del estudiante. Se observa, entonces, que existen elementos que propasan la dimensión de la motivación del profesor, la convicción en el profesor y en la propia actividad docente.

El profesor, en su práctica, hace una amalgama para lidiar con los contenidos en la enseñanza y con sus actividades prácticas. En el caso del profesor de Geografía, esa mezcla dice respecto al objetivo de desarrollo del pensamiento geográfico. En ese sentido, los momentos de encuentro en grupos cooperativos han sido considerados como una posibilidad más que el profesor tiene para la reflexionar entre sus pares y para encontrar caminos conjuntos que permitan el desarrollo de ese pensamiento en los estudiantes. En esos momentos, es común el desahogo de los profesores sobre problemas de su profesión, pero también ocurren nuevas percepciones entre los participantes, en sus narrativas, como posibles salidas para superar esos problemas en diferentes escuelas. En los grupos, la proximidad entre los profesores ha permitido observar mejor los principales desafíos de la profesión y buscar propuestas de cambios.

\section{BIBLIOGRAFÍA}

Carvalho, J. M. de. (2002). Cidadania no Brasil: o longo Caminho. Rio de Janeiro: Civilização Brasileira.

Cavalcanti, L. S. (2014). A metrópole em foco no ensino de Geografia: o que/ para que/ para quem ensinar? In, F. M. A. Paula; L. S. L. S. Cavalcanti \& V. C. Souza (Eds.), Ensino de Geografia e metrópole (pp. 27- 41). Goiânia: Gráfica e Editora América.

Cavalcanti, L. S. (2017). Ensino de Geografia e demandas contemporâneas: práticas e formação docentes. In A. O. Alves \& A. M. K. Khaoul. (Coords.), A geografia no 
cenário das políticas públicas educacionais. (pp. 15-32). Goiânia: C\&A Alfa \& Comunicação.

Cavalcanti, L. S. (2019). Pensar pela geografia: ensino e relevância social. Goiânia: C\&A Alfa Comunicação.

Cavalcanti, L. S. (2020). Formar para a vida urbana cidadã como meta para o professor de Geografia: aportes teóricos e ensinamentos da experiência. AGALI Journal, 10, 45-64.

Díaz, E. G.; Porlán, R. \& Navarro, E. (2017). Los fines y los contenidos de enseñanza. In: R. Porlán (Eds.), Ensenãnza universitária: como mejorarla (pp. 55-72). Madrid: Morata.

Ferreira, A. A. \& Silva, B. D. (2014). Comunidade de prática on-line: uma estratégia para o desenvolvimento profissional dos professores de História. Educação em Revista, 30, 37-64.https://doi.org/10.1590/S0102-46982014000100003

Fiorentini, D. (2010a). Desenvolvimento Profissional e Comunidades Investigativas. In A. Dalben; J. Diniz; L. Leal \& L. Santos (Eds.), Convergências e tensões no campo da formação e do trabalho docente (pp. 570-590). Belo Horizonte: Autêntica.

Fiorentini, D. (2010b). Grupo de sábado: uma história de reflexão, investigação e escrita sobre a prática escolar em matemática. In D. Fiorentini \& Em M. Cristovão (Eds.), História e Investigações delem aulas de matemática (pp. 13-36). Campinas, SP: Editora alínea.

Imbernón, F. (2010). Formação docente e profissional: forma-se para a mudança e a incerteza. $8^{\mathrm{a}}$ ed. São Paulo: Cortez.

Gomes, P. C. C. (2013). O lugar do olhar: elementos para uma geografia da visibilidade. Rio de Janeiro: Bertrand Brasil.

Manrique, A. L. (2017). Uma síntese de pesquisas sobre aprendizagem docente. In A. L. Manrique; S. M. Lacerda \& W. R. Silva (Eds.), Pesquisa em rede colaborativa: aprendizagens docentes (pp. 7-16). São Paulo: Editora Livraria da Física.

Marcelo Garcia, C. (1999). Formação de professores: para uma mudança educativa. Porto: Porto editora.

Montero, L. (2001). A construção do conhecimento profissional docente. Lisboa: Instituto Piaget.

Moreira, R. (2007). Pensar e ser em Geografia. São Paulo: Ed. Contexto.

Santos, M. (1996.) Por uma geografia cidadã: por uma espitemologia da existência. Boletim Gaúcho de Geografia, 21, 7-14.

Shulman, L. S. (2005) Conocimiento y enseñanza: fundamentos de la nueva reforma. Profesorado. Revista de Currículum y formación del professorado, 9, 2, 1-30. 
Tanaka, A. L. F. \& Passos, L. F. (2017). Tempos de aprender... Tempos de compartilhar: professores experientes e professores iniciantes num grupo colaborativo. In A. L. Manrique; S. M. Lacerda \& W. R. Silva (Eds.), Pesquisa em rede colaborativa: aprendizagens docentes (pp. 97-113). São Paulo: Editora Livraria da Física. 